\title{
Pre- and postoperative left atrial and ventricular volumetric and deformation analyses in severe aortic regurgitation
}

Jonas Jenner ${ }^{1,2,3^{*}}$ (D), Ali llami ${ }^{1,3}$, Johan Petrini ${ }^{1,2}$, Per Eriksson ${ }^{4}$, Anders Franco-Cereceda ${ }^{1,5}$, Maria J. Eriksson ${ }^{1,3+}$ and Kenneth Caidahl $1^{1,6+}$

\begin{abstract}
Background: The impact of volume overload due to aortic regurgitation (AR) on systolic and diastolic left ventricular (LV) indices and left atrial remodeling is unclear. We assessed the structural and functional effects of severe AR on LV and left atrium before and after aortic valve replacement.

Methods: Patients with severe AR scheduled for aortic valve replacement $(n=65)$ underwent two- and threedimensional echocardiography, including left atrial strain imaging, before and 1 year after surgery. A control group was selected, and comprised patients undergoing surgery for thoracic aortic aneurysm without aortic valve replacement $(n=20)$. Logistic regression analysis was used to assess predictors of impaired left ventricular functional and structural recovery, defined as a composite variable of diastolic dysfunction grade $\geq 2, E F<50 \%$, or left ventricular end-diastolic volume index above the gender-specific normal range.

Results: Diastolic dysfunction was present in $32 \%$ of patients with AR at baseline. Diastolic LV function indices and left atrial strain improved, and both left atrial and LV volumes decreased in the AR group following aortic valve replacement. Preoperative left atrial strain during the conduit phase added to left ventricular endsystolic volume index for the prediction of impaired LV functional and structural recovery after aortic valve replacement (model $p<0.001$, accuracy $70 \%$; addition of left atrial strain during the conduit phase to endsystolic volume index $p=0.006$ ).

Conclusions: One-third of patients with severe AR had signs of diastolic dysfunction. Aortic valve surgery reduced LV and left atrial volumes and improved diastolic indices. Left atrial strain during the conduit phase added to the well-established left ventricular end-diastolic dimension for the prediction of impaired left ventricular functional and structural recovery at follow-up. However, long-term follow-up studies with hard endpoints are needed to assess the value of left atrial strain as predictor of myocardial recovery in aortic regurgitation.
\end{abstract}

Keywords: Aortic valve replacement, Left atrial function, Transthoracic echocardiography, Left atrial strain

\footnotetext{
* Correspondence: jonas.jenner@ki.se

${ }^{+}$Maria J. Eriksson and Kenneth Caidahl contributed equally to this work.

'Department of Molecular Medicine and Surgery, Karolinska Institutet,

Stockholm, Sweden

${ }^{2}$ Department of Clinical Physiology, Södersjukhuset, Stockholm, Sweden

Full list of author information is available at the end of the article
}

(c) The Author(s). 2021 Open Access This article is licensed under a Creative Commons Attribution 4.0 International License, which permits use, sharing, adaptation, distribution and reproduction in any medium or format, as long as you give appropriate credit to the original author(s) and the source, provide a link to the Creative Commons licence, and indicate if changes were made. The images or other third party material in this article are included in the article's Creative Commons licence, unless indicated otherwise in a credit line to the material. If material is not included in the article's Creative Commons licence and your intended use is not permitted by statutory regulation or exceeds the permitted use, you will need to obtain permission directly from the copyright holder. To view a copy of this licence, visit http://creativecommons.org/licenses/by/4.0/ The Creative Commons Public Domain Dedication waiver (http://creativecommons.org/publicdomain/zero/1.0/) applies to the data made available in this article, unless otherwise stated in a credit line to the data. 


\section{Background}

Severe chronic aortic regurgitation (AR) is characterized by volume overload of the left ventricle. This induces structural and functional left ventricular (LV) alterations, which have a negative prognostic impact even in patients who are asymptomatic [1-3].

Despite the emerging potentials of transcatheter valve replacement, the treatment of choice for severe AR is still aortic valve replacement (AVR), with intervention timing based on the presence of symptoms or evidence of increased LV dimensions or systolic dysfunction [4-6]. Recent studies indicate that the cutoff values used in current guidelines might need to be reconsidered $[7,8]$, and additional measures such as strain imaging for LV have proven useful $[9,10]$. Thus, most studies of chronic AR have focused on LV dimensions and systolic function, to intervene while it is still possible to achieve full functional myocardial recovery and optimal life expectancy. However, chronic volume overload in AR also leads to decreased LV relaxation, increased stiffness, and, subsequently increased filling pressures (i.e., diastolic LV dysfunction) [11]. LV diastolic property alterations influence left atrial (LA) size. LA enlargement is an important marker of LV diastolic dysfunction (DD) and is incorporated in echocardiographic DD assessment algorithms [12]. It is still unclear if LA function may be a more sensitive marker of early myocardial impairment and volume overload in chronic severe AR [11].

LA function, in terms of the reservoir, conduit, and contraction phases, can be assessed quantitatively by measuring left atrial strain (LAS) using speckle-tracking echocardiography [13]. LAS has emerged as a useful diagnostic and prognostic parameter in various cardiovascular conditions, including valvular heart disease [14, 15]. However, data on LA phasic function in patients with AR are limited, especially regarding whether this is altered after valve surgery [16]. Furthermore, the value of baseline LA function variables for predicting myocardial recovery after AVR has not been fully elucidated.

We hypothesized that the reduction in LV overload following aortic valve surgery would improve markers of diastolic LV function and LA phasic function. To address this, we performed a prospective, longitudinal study of patients with chronic, severe AR without coronary artery disease using three-dimensional (3D) and twodimensional (2D) echocardiography, including speckletracking echocardiography. We aimed to assess the effects of AR-related LV volume overload on LV and LA structure and function before, and 1 year after, aortic valve surgery. As a secondary aim, we sought to evaluate whether preoperative LA remodeling and functional changes could predict postoperative LV dysfunction.

\section{Methods}

\section{Study group}

The study group was recruited from a prospective, observational study at Karolinska University Hospital in Stockholm, Sweden, conducted from 2007 to 2013. The study included consecutive adult patients free from coronary artery disease (i.e., without symptoms or epicardial stenoses) who were undergoing elective open-heart surgery for aortic valve disease or thoracic aortic aneurysm (TAA) [17]. From this cohort, 101 patients with chronic severe AR and a transvalvular mean pressure gradient < $20 \mathrm{mmHg}$, with complete 2D and 3D echocardiograms performed pre- and postoperatively, were eligible for inclusion. Exclusion criteria were two or more adjacent LV segments not visualized in either echocardiogram $(n=26)$ or atrial fibrillation $(n=5)$. Five additional cases were excluded for technical reasons (data stored in incompatible format); the final study group consisted of 65 patients.

A control group was included comprising 20 consecutive patients with TAA and no significant aortic valve disease, defined as no or mild AR and transvalvular mean pressure gradient $<20 \mathrm{mmHg}$, who underwent surgery for TAA during the same period.

\section{Transthoracic echocardiography}

Comprehensive transthoracic 2D and 3D echocardiographic examinations were performed 1 week or less before surgery and at a follow-up visit 1 year after surgery, using commercially available equipment (Philips iE33 or Epic 7; Philips Medical Systems, Bothell, WA, USA). 3D data were acquired over four or seven cardiac cycles, generating full volume datasets. The echocardiograms were performed following current recommendations by two experienced sonographers [18]. Data were stored for offline analysis.

2D echocardiographic data were analyzed using dedicated software (IntelliSpace Cardiovascular 2.3; Philips Medical Systems Nederland B.V., Eindhoven, The Netherlands). LV dimensions and wall thickness were measured in the parasternal long-axis view. LA volume was calculated using the biplane method of disks and indexed to body surface area (BSA). LV stroke work (in gram-meters) was calculated as $\mathrm{SW}=\mathrm{BP}_{\mathrm{s}} \times \mathrm{SV} \times 0.014$, where $\mathrm{BP}_{\mathrm{s}}$ is systolic blood pressure and $\mathrm{SV}$ is 3D echocardiographic stroke volume [19].

Diastolic function variables were acquired and analyzed according to the current guidelines, and DD was defined by incorporating the ratio between early and late diastolic filling velocities (E/A ratio), the ratio between mitral early filling velocity and average annular tissue velocity (E/e' ratio), tricuspid regurgitation velocity, and LA volume index (LAVi). Patients were classified into three categories: (i) no or grade $1 \mathrm{DD}$, (ii) grade $2 \mathrm{DD}$, 
and (iii) grade 3 DD [12]. Cases that could not be assigned a DD grade were deemed indeterminate.

LV global longitudinal strain (GLS) was measured using dedicated software (QLab 10.7; Philips Medical Systems Nederland B.V.). The LV wall was traced automatically throughout the cardiac cycle using a speckletracking algorithm. The region of interest was manually adjusted if needed. GLS was calculated by averaging the peak systolic longitudinal strain of 17 segments and is expressed as an absolute percentage $(|\%|)$.

LAS was calculated using dedicated software (TomTec-Arena, 2D CPA; TomTec Imaging Systems GmbH, Unterschleißheim, Germany). Two points were placed at the mitral annulus and a third at the LA roof. The LA wall was then automatically tracked through the cardiac cycle, generating an atrial GLS curve. If needed, delineation was corrected manually at end-systole and end-diastole. The zero-strain reference point was set to the end-diastolic frame following mitral valve closure (Fig. 1). The LAS curve was divided into three phases: (i) a reservoir phase (LASr); (ii) a conduit phase (LAScd); and (iii) a contraction phase (LASct) [20]. Results are reported as the means of measurements obtained from four- and twochamber views.

Intraobserver variabilities in LASr, LASct, and LAScd measurements were assessed by repeated measurements in the same images acquired from 20 randomly selected cases by one blinded observer (A.I.). To determine the interobserver variability, the measurements were repeated in the same images by a second observer (J.J.) who was blinded to the results obtained by the first observer. Agreement was assessed by calculating the intraclass correlation coefficient (ICC). ICC values for intraobserver agreement of the LASr, LAScd, and LASct estimates were 0.83 (95\% CI 0.62-0.93), 0.87 (95\% CI 0.69-0.95), and 0.69 (95\% C.I. 0.35-0.87), respectively. ICC values for interobserver agreement were $0.76(95 \%$ CI $0.43-0.90), 0.85$ (95\% CI $0.66-0.94)$, and 0.76 (95\% CI 0.19-0.92), respectively.

LV volume and mass measurements in $3 \mathrm{D}$ datasets were performed using dedicated software (QLab 10.7, as above). The endocardial surface was outlined using a semi-automated contour detection algorithm, yielding the end-diastolic volume (EDV) and end-systolic volume (ESV). Papillary muscles and trabeculations were included in the cavity. Stroke volume (SV) was calculated from EDV-ESV, and the ejection fraction (EF) was calculated as EDV-ESV/EDV $\times 100 \%$.

LV mass was calculated using the biplane method of disks in four- and two-chamber views obtained from 3D datasets. The endocardium and epicardium were traced manually in each view, with the papillary muscles included in the LV cavity. The myocardial mass was then calculated by the software. Measurements were made at end-diastole and end-systole, and the LV mass was estimated as the mean of the two measurements and indexed to BSA.

\section{Surgical procedures}

All patients were operated on via a midline sternotomy using cardiopulmonary bypass. In the AR group, AVR was performed using biological valve prostheses $(n=16)$, mechanical valve prostheses $(n=15)$, aortic root

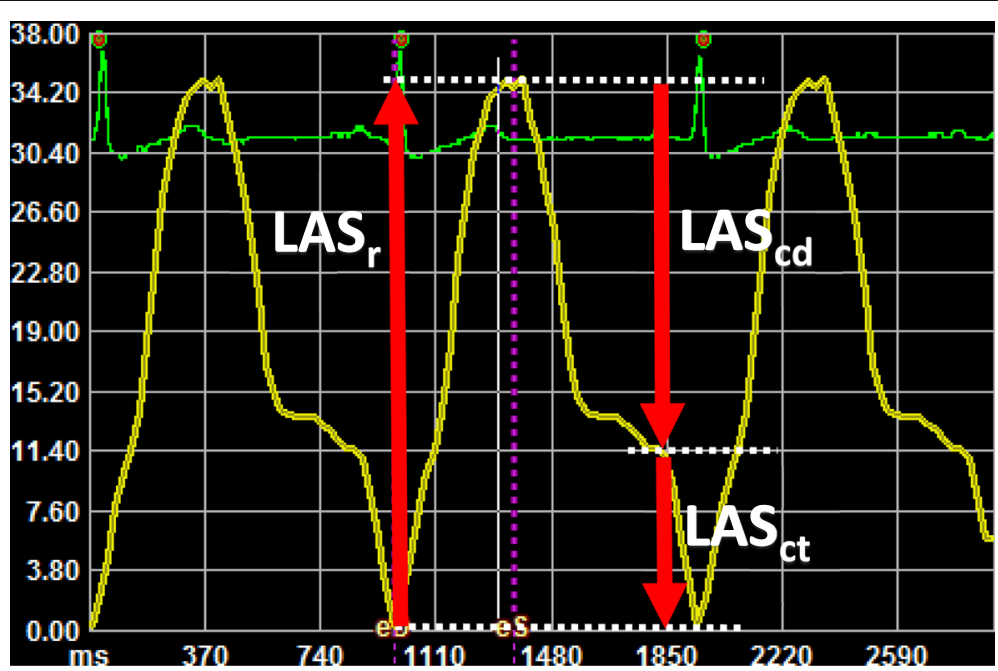

Fig. 1 Example of a left atrial strain (LAS) curve (yellow line). The $x$ axis is time, and the y axis represents strain as a percentage. The ECG recording is shown in green for reference. The zero-strain reference is set at end-diastole. Dashed white lines represent strain values at end-diastole (0), end-systole, and the beginning of atrial contraction. Red arrows demonstrate the left atrial strain components. LASr, left atrial reservoir phase; LAScd, left atrial conduit phase; LASct, left atrial contraction phase 
bioprostheses $(n=5)$, or aortic root replacement with a mechanical composite graft $(n=5)$. Isolated aortic valve repair was performed in 21 patients, while 3 underwent aortic root replacement with reimplantation of the native valve. The group with isolated TAA received synthetic tubular aortic grafts but no valve surgery.

\section{$\mathrm{N}$-terminal pro-B-type natriuretic peptide (NTproBNP) analysis}

Plasma samples were analyzed using a multiplex immunoassay (Olink Cardiovascular III; Olink Proteomics, Uppsala, Sweden). NTproBNP concentration is reported as normalized protein expression (NPX) units. NPX is a $\log _{2}$ scale; hence, an increase of 1 NPX unit means a doubling of protein concentration.

\section{Statistical analysis}

Continuous variables are expressed as the mean $\pm \mathrm{SD}$ or the median and interquartile range (IQR) depending on the data distribution. A $p$ value $<0.05$ was considered statistically significant. Correlations between continuous variables were assessed by calculating Pearson's correlation coefficient $r$. Between-groups comparisons of the means of continuous variables were performed using Student's $t$-test and analysis of variance (ANOVA). Bonferroni correction was used to adjust for multiple comparisons. Differences between unrelated samples of ordinal data were assessed using the Mann-Whitney $U$ test. Relationships between categorical variables were assessed using the chi-squared or Fisher's exact test.

Simple and multiple logistic regression analyses were performed to assess predictors of impaired LV functional and structural recovery, which was defined as a composite variable comprising one or more of the following echocardiographic characteristics at the follow-up examination: $\mathrm{EF}<50 \%, \mathrm{DD}$ grade $\geq 2$, and EDV index (EDVi) greater than the gender-specific normal range [18]. Baseline variables with $p$ values $<0.10$ in simple logistic regressions were assessed for collinearity and entered pairwise in multiple logistic regression models. The reason for restricting the models to two variables at a time was the risk of overfitting. All combinations were tested, and classification tables and Nagelkerke $R^{2}\left(\mathrm{R}_{\mathrm{N}}^{2}\right)$ values were assessed to rank the models with respect to their predictive performances. The likelihood ratio test (LRT) was used to determine whether a variable contributed significantly to the model. Discriminatory ability was assessed by receiver operating characteristic (ROC) analysis and shown as area under the ROC curve (AUC). Statistical analyses were performed using IBM SPSS Statistics (version 26; IBM Corp., Armonk, NY, USA).

\section{Results}

The baseline characteristics of the AR and control groups are listed in Table 1. Patients with AR had higher NTproBNP levels, and 75\% had a New York Heart Association (NYHA) class $>$ I, compared with $10 \%$ of the controls $(p<0.001)$. At the follow-up examination, no patient undergoing valve replacement, and 3 patients with valve repair, had moderate residual AR; none of which warranted reoperation. Of these 3 patients, one had $\mathrm{EF}<50 \%$. There were no cases of atrial fibrillation at the follow-up examinations.

Table 1 Preoperative demographic data

\begin{tabular}{|c|c|c|c|}
\hline & Aortic regurgitation $(n=65)$ & Controls $(n=20)$ & $p$ value \\
\hline Age (years) median (IQR) & $54(46-63)$ & $59(49-68)$ & 0.086 \\
\hline Male (n) & $56(86 \%)$ & $11(55 \%)$ & 0.009 \\
\hline Body surface area $\left(\mathrm{m}^{2}\right)$ & $2.00 \pm 0.19$ & $1.96 \pm 0.20$ & 0.46 \\
\hline Body mass index $\left(\mathrm{kg} / \mathrm{m}^{2}\right)$ & $26.1 \pm 4.0$ & $26.3 \pm 3.6$ & 0.91 \\
\hline Systolic blood pressure $(\mathrm{mmHg})$ & $145 \pm 16$ & $134 \pm 15$ & 0.011 \\
\hline Diastolic blood pressure $(\mathrm{mmHg})$ & $70 \pm 11$ & $84 \pm 9$ & $<0.001$ \\
\hline Diabetes (n) & $1(1.5 \%)$ & $1(5 \%)$ & 0.77 \\
\hline Hypertension (n) & $30(46 \%)$ & $10(50 \%)$ & 0.80 \\
\hline NYHA functional class & & & $<0.001$ \\
\hline । & $16(25 \%)$ & $18(90 \%)$ & \\
\hline$\|$ & $39(60 \%)$ & $2(10 \%)$ & \\
\hline III & $10(15 \%)$ & 0 & \\
\hline IV & 0 & 0 & \\
\hline NTproBNP (NPX units) & $4.4 \pm 1.7$ & $3.52 \pm 1.3$ & 0.043 \\
\hline Bicuspid aortic valve (n) & $38(58 \%)$ & $9(45 \%)$ & 0.29 \\
\hline
\end{tabular}

IQR, Interquartile range, NPX units, normalized protein expression units ( $\log _{2}$ scale), NTproBNP, N-terminal pro-B-type natriuretic peptide; NYHA, New York Heart Association 
Table 2 Preoperative and follow-up values for left ventricular and left atrial dimensions, volumes, and function

\begin{tabular}{|c|c|c|c|c|c|c|c|}
\hline & \multicolumn{3}{|c|}{ Aortic regurgitation $(n=65)$} & \multicolumn{3}{|c|}{ Controls $(n=20)$} & \multirow{2}{*}{$\begin{array}{l}\text { Baseline comparison } \\
p \text { value }\end{array}$} \\
\hline & Baseline & Follow-up & $p$ value & Baseline & Follow-up & $p$ value & \\
\hline \multicolumn{8}{|l|}{ Left ventricular indices } \\
\hline End-diastolic diameter (mm) & $62.6 \pm 6$ & $49.3 \pm 6$ & $<0.001$ & $48.5 \pm 3$ & $46.2 \pm 4$ & 0.005 & $<0.001$ \\
\hline End-systolic diameter (mm) & $44.0 \pm 7$ & $34.4 \pm 5$ & $<0.001$ & $33.1 \pm 4$ & $32.5 \pm 4$ & 0.80 & $<0.001$ \\
\hline Interventricular septum (mm) & $11.7 \pm 2.0$ & $12.2 \pm 1.7$ & 0.004 & $11.3 \pm 2.0$ & $11.4 \pm 1.7$ & 0.71 & 0.48 \\
\hline Posterior wall thickness (mm) & $10.3 \pm 1.4$ & $9.5 \pm 1.8$ & 0.003 & $8.9 \pm 1.7$ & $9.3 \pm 1.6$ & 0.29 & $<0.001$ \\
\hline End-diastolic volume (mL) & $224 \pm 62$ & $132.4 \pm 36$ & $<0.001$ & $109 \pm 20$ & $111 \pm 23$ & 0.25 & $<0.001$ \\
\hline End-diastolic volume index $\left(\mathrm{mL} / \mathrm{m}^{2}\right)$ & $113 \pm 30$ & $66 \pm 17$ & $<0.001$ & $56 \pm 9$ & $57 \pm 10$ & 0.40 & $<0.001$ \\
\hline End-systolic volume (mL) & $103 \pm 41$ & $57 \pm 18$ & $<0.001$ & $43 \pm 8$ & $45 \pm 11$ & 0.25 & $<0.001$ \\
\hline End-systolic volume index $\left(\mathrm{mL} / \mathrm{m}^{2}\right)$ & $52 \pm 20$ & $28 \pm 8$ & $<0.001$ & $22 \pm 4$ & $23 \pm 5$ & 0.41 & $<0.001$ \\
\hline Stroke volume $(\mathrm{mL})$ & $121 \pm 31$ & $76 \pm 22$ & $<0.001$ & $65 \pm 14$ & $66 \pm 15$ & 0.41 & $<0.001$ \\
\hline Ejection fraction (\%) & $55 \pm 7.3$ & $57 \pm 7.1$ & $0.027^{\mathrm{a}}$ & $60 \pm 4$ & $60 \pm 7$ & 0.79 & $<0.001$ \\
\hline Global longitudinal strain $(|\%|)$ & $19.0 \pm 3.0$ & $19.4 \pm 2.5$ & 0.34 & $19.9 \pm 2$ & $19.6 \pm 2$ & 0.54 & 0.24 \\
\hline Left ventricular mass index $\left(\mathrm{g} / \mathrm{m}^{2}\right)$ & $81 \pm 19$ & $62 \pm 16$ & $<0.001$ & $48 \pm 11$ & $48 \pm 11$ & 0.87 & $<0.001$ \\
\hline \multicolumn{8}{|l|}{ Diastolic function indices } \\
\hline Mitral E velocity $(\mathrm{m} / \mathrm{s})$ & $0.76 \pm 0.2$ & $0.73 \pm 0.2$ & 0.17 & $0.70 \pm 0.2$ & $0.76 \pm 0.2$ & 0.14 & 0.23 \\
\hline Mitral A velocity $(\mathrm{m} / \mathrm{s})$ & $0.58 \pm 0.2$ & $0.59 \pm 0.2$ & 0.84 & $0.63 \pm 0.2$ & $0.66 \pm 0.2$ & 0.38 & 0.30 \\
\hline Mitral E/A ratio & $1.5 \pm 0.7$ & $1.3 \pm 0.5$ & 0.063 & $1.3 \pm 0.8$ & $1.2 \pm 0.6$ & 0.93 & 0.17 \\
\hline Mitral E-wave Deceleration time (ms) & $205 \pm 57$ & $238 \pm 52$ & 0.001 & $212 \pm 44$ & $232 \pm 52$ & 0.11 & 0.66 \\
\hline Septal e' $(\mathrm{m} / \mathrm{s})$ & $0.074 \pm 0.02$ & $0.075 \pm 0.02$ & 0.85 & $0.066 \pm 0.02$ & $0.070 \pm 0.02$ & 0.46 & 0.11 \\
\hline Lateral e' (m/s) & $0.097 \pm 0.03$ & $0.12 \pm 0.03$ & 0.005 & $0.087 \pm 0.02$ & $0.10 \pm 0.01$ & 0.071 & 0.13 \\
\hline Mitral average E/e' ratio & $9.3 \pm 3$ & $8.0 \pm 3$ & 0.012 & $10.2 \pm 3$ & $9.1 \pm 2$ & 0.31 & 0.30 \\
\hline Tricuspid regurgitation velocity (m/s) & $2.5 \pm 0.3$ & $2.3 \pm 0.4$ & 0.080 & $2.3 \pm 0.2$ & $2.3 \pm 0.2$ & 0.33 & $0.047^{a}$ \\
\hline \multicolumn{8}{|l|}{ Diastolic dysfunction grade } \\
\hline 0 or 1 & 39 (60\%) & $55(85 \%)$ & 0.003 & $20(100 \%)$ & 18 (90\%) & 0.30 & 0.002 \\
\hline 2 & $6(9 \%)$ & $2(3 \%)$ & & 0 & $1(5 \%)$ & & \\
\hline 3 & 15 (23\%) & $5(8 \%)$ & & 0 & 0 & & \\
\hline Indeterminate & $5(8 \%)$ & $3(4 \%)$ & & 0 & $1(5 \%)$ & & \\
\hline \multicolumn{8}{|l|}{ Left atrial indices } \\
\hline Left atrial volume index $\left(\mathrm{ml} / \mathrm{m}^{2}\right)$ & $38.6 \pm 12$ & $32.2 \pm 11$ & $<0.001$ & $26.8 \pm 8$ & $27.4 \pm 8$ & 0.70 & $<0.001$ \\
\hline LASr (\%) & $26.3 \pm 6.7$ & $29.0 \pm 6.2$ & 0.012 & $25.7 \pm 6.0$ & $25.8 \pm 4.7$ & 0.92 & 0.86 \\
\hline LAScd (\%) & $15.6 \pm 6.1$ & $14.0 \pm 4.5$ & $0.037^{\mathrm{a}}$ & $12.1 \pm 3.5$ & $11.9 \pm 3.7$ & 0.28 & 0.081 \\
\hline LASct (\%) & $11.0 \pm 4.7$ & $15.3 \pm 5.5$ & $<0.001$ & $12.8 \pm 5.6$ & $13.8 \pm 4.2$ & 0.43 & $0.049^{\mathrm{a}}$ \\
\hline
\end{tabular}

${ }^{a}$ Non-significant after Bonferroni correction at $\mathrm{a}=0.017$; $e^{\prime}$, tissue doppler-derived mitral annular early diastolic velocity, LAScd Left atrial strain conduit phase, LASct Left atrial strain contraction phase, LASr Left atrial strain reservoir phase

LV size and function before and after surgery

At baseline, the patients with AR had increased LV dimensions and volumes, larger SV, increased LV mass index (LVMi), and lower EF compared with the controls, whereas no difference in GLS was found (Table 2). In patients with AR, the NTproBNP measure was correlated with GLS $(r=-0.43, p=0.001)$, but not with EF $(p=0.076)$.

At follow-up, decreases were observed in LV dimensions and volumes, SV, wall thickness, and LVMi, while the EF had increased in patients with AR; these variables were unchanged in the control group. The change in GLS from baseline to follow-up in patients with AR was correlated with the changes in LASr $(r=0.35, p=0.007)$ and LAScd ( $r=0.34, p=0.009$; Fig. 2 ).

\section{LV diastolic function and LA function before and after surgery}

At baseline, patients with AR had larger LAVi, a higher prevalence of DD grade 2 or 3, and lower LASct measures compared with the control group (Table 2). NTproBNP correlated with LASr $(r=-0.43, p=0.001)$, 


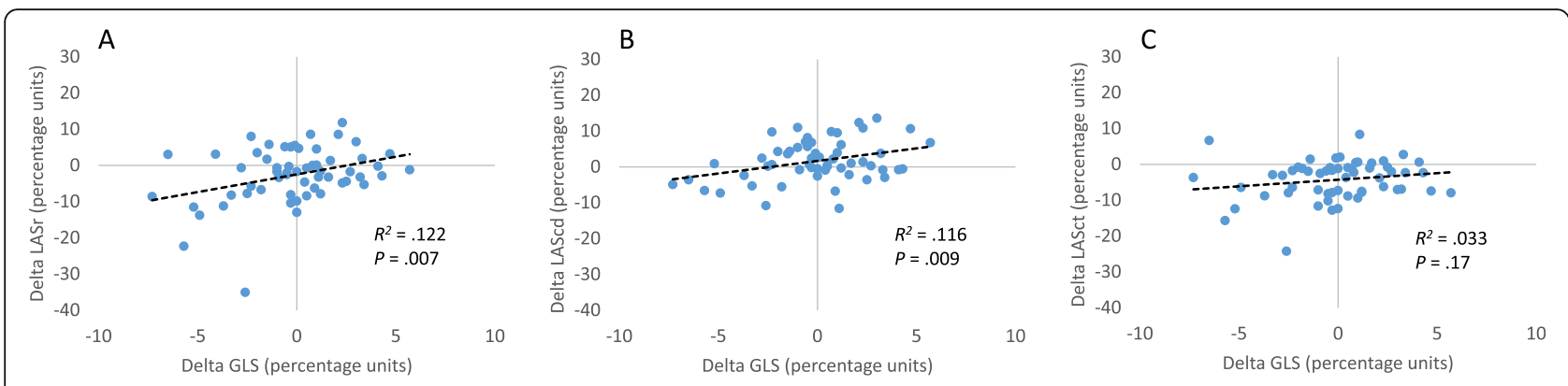

Fig. 2 Changes between baseline and follow-up in left ventricular and left atrial strain indices in patients with aortic regurgitation. Left ventricular global longitudinal strain (GLS) vs. left atrial strain (LAS) reservoir phase (a), conduit phase (b), and contraction phase (c). GLS, Global longitudinal strain; LASr, left atrial reservoir phase; LAScd, left atrial conduit phase; LASct, left atrial contraction phase

LAScd $(r=-0.39, p=0.002)$, and LAVi $(r=0.47, p<$ 0.001).

At follow-up in the AR group, indices of LV diastolic function improved, LAVi decreased, and increases were seen in the LASr and LASct estimates. No changes were observed in the control group in diastolic function, LAVi, or LAS variables (Table 2; Fig. 3).

Patients with AR classified as DD grades 2 or 3 had lower LASr, LASct, and LASct/LASr ratios, and higher NTproBNP levels compared with patients with AR in DD grades 0 or 1 and with controls (Table 3 ).

\section{Determinants of impaired LV functional and structural recovery}

At follow-up, 27 patients fulfilled one or more of the composite outcome criteria: $\mathrm{EF}<50 \%$, DD grade $\geq 2$, EDVi above the normal range according to current guidelines $\left(>79 \mathrm{~mL} / \mathrm{m}^{2}\right.$ for men and $<71 \mathrm{~mL} / \mathrm{m}^{2}$ for women) [18]. Baseline variables were analyzed using simple logistic regression (Table 4). The variable that best predicted impaired LV functional and structural recovery in the unadjusted analysis was end-systolic volume index (ESVi) (accuracy 69\%; OR 1.07, $p=0.001$ ).
Variables with $p$ values $<0.10$ in simple regressions were tested for collinearity and subsequently added to ESVi in multiple regression analyses. The model that best predicted impaired LV functional and structural recovery was LAScd combined with ESVi (accuracy 70\%, Table 5). LAScd was the only variable that added significantly to ESVi to predict the outcome variable (LRT $p=0.006$ ); the model with LAScd and ESVi had greater discriminatory ability compared with ESVi alone (AUC 0.83 vs. $0.78, p=0.046)$.

\section{Discussion}

To the best of our knowledge, this is the first follow-up study to assess changes in LA phasic function following AVR in patients with severe AR. Our results demonstrate that patients with AR have larger LAVi and reduced LASct compared with controls. One year after surgery, LAVi and LAScd had decreased, LA strain during LASr and LASct had increased, and an improvement was seen in diastolic function indices and consequently DD grade. These findings suggest that the volume overload imposed by AR affects not only LV volume and mass and systolic function but also LV diastolic
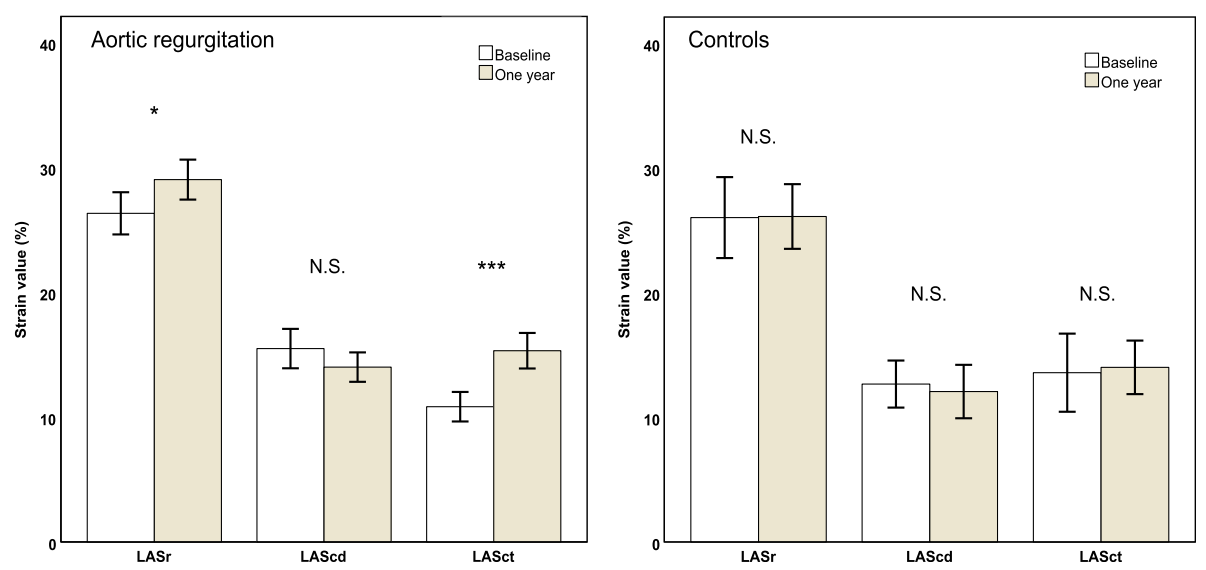

Fig. 3 Left atrial strain (LAS) components at baseline and 1 year after surgery. Error bars represent $95 \%$ confidence intervals. LASr, left atrial reservoir phase; LAScd, left atrial conduit phase; LASct, left atrial contraction phase. ${ }^{*} p \leq 0.05,{ }^{* * *} p \leq 0.001$, N.S. $p>0.05$ 
Table 3 Left atrial strain and NTproBNP levels in patients with aortic regurgitation (based on diastolic dysfunction grade) and controls

\begin{tabular}{lllll}
\hline & Aortic regurgitation $(\boldsymbol{n = 6 0 )}$ & & $\begin{array}{l}\text { Controls } \\
(\boldsymbol{n}=\mathbf{2 0})\end{array}$ & $\begin{array}{l}\text { Overall } \\
\boldsymbol{p} \text { value }\end{array}$ \\
\cline { 2 - 5 } & DD grades $\mathbf{0}$ or $\mathbf{1}(\boldsymbol{n}=\mathbf{3 9 )}$ & DD grades $\mathbf{2}$ or $\mathbf{3}(\boldsymbol{n}=\mathbf{2 1})$ & $25.7 \pm 6.0$ & 0.030 \\
\hline LASr (\%) & $27.9 \pm 5.1^{*}$ & $23.8 \pm 7.8$ & $12.1 \pm 3.5$ & 0.26 \\
LAScd (\%) & $15.3 \pm 6.8$ & $15.8 \pm 6.8$ & $12.8 \pm 5.6$ & $<0.001$ \\
LASct (\%) & $12.6 \pm 4.2^{*}$ & $8.0 \pm 3.7^{\dagger}$ & $0.50 \pm 0.14$ & 0.001 \\
LASct/LASr ratio & $0.47 \pm 0.15^{*}$ & $0.34 \pm 0.13^{\dagger}$ & $3.53 \pm 1.31$ & 0.002 \\
NTproBNP (NPX units) & $3.79 \pm 1.61^{*}$ & $5.03 \pm 1.54^{\dagger}$ &
\end{tabular}

$D D$ Diastolic dysfunction, LASCd Left atrial conduit phase, LASct Left atrial contraction phase, LASr Left atrial reservoir phase, NPX units Normalized protein expression units ( $\log _{2}$ scale), NTproBNP N-terminal pro-B-type natriuretic peptide. ${ }^{*} p<0.05$ for the comparison between DD grades 0 or 1 and DD grades 2 or 3 , ${ }^{+} p<0.05$ for the comparison between DD grades 2 or 3 and the control group

properties and LA structure and function. We also found that preoperative LA function, in terms of LAScd, added to the established measure of end-systolic LV dimension for the prediction of impaired LV functional and structural recovery following AVR for severe AR.

\section{Systolic LV function}

Severe AR leads to increased preload of the LV, inducing progressive dilation, which in turn increases wall stress and, over time, can cause structural myocardial changes and depressed ventricular systolic function [21]. Thus, LV size and EF are well-established predictors of disease

Table 4 Simple logistic regression analyses of predictors of impaired LV functional and structural recovery in aortic regurgitation patients undergoing aortic valve surgery

\begin{tabular}{llll}
\hline & Odds ratio $(95 \%$ Cl) & $\boldsymbol{p}$ value & $\boldsymbol{R}_{\mathbf{N}}{ }^{2}$ \\
\hline Age & $0.96(0.92-1.00)$ & 0.045 & 0.09 \\
Gender & $0.6(0.14-2.67)$ & 0.51 & 0.10 \\
EDVi & $1.04(1.02-1.07)$ & 0.001 & 0.29 \\
ESVi & $1.07(1.03-1.12)$ & 0.001 & 0.28 \\
EF & $0.93(0.86-1.01)$ & 0.091 & 0.07 \\
GLS & $0.9(0.75-1.08)$ & 0.26 & 0.03 \\
Stroke work & $1.01(1-1.02)$ & 0.040 & 0.10 \\
DD grade $\leq 1$ vs. $\geq 2$ & $0.62(0.2-1.89)$ & 0.40 & 0.02 \\
E/e' & $0.94(0.82-1.08)$ & 0.40 & 0.02 \\
LAVi & $0.99(0.94-1.03)$ & 0.53 & 0.01 \\
LASr & $1.1(1-1.2)$ & 0.059 & 0.05 \\
LAScd & $1.15(1.03-1.28)$ & 0.010 & 0.17 \\
LASct & $0,95(0,84-1,07)$ & 0.36 & 0.02 \\
NYHA I vs. II-III & $1.68(0.52-5.43)$ & 0.38 & 0.02 \\
NTproBNP & $0.98(0.72-1.33)$ & 0.91 & 0.0
\end{tabular}

$C /$ Confidence interval, $D D$ Diastolic dysfunction, $E / e^{\prime}$ ratio between mitral early filling velocity and annular tissue velocity, EF Ejection fraction, EDVi Enddiastolic volume index, ESVi End-systolic volume index, GLS Left ventricular global longitudinal strain, LASCd Left atrial strain conduit phase, LASCt Left atrial strain contraction phase, LASr Left atrial strain reservoir phase, LAVi Left atrial volume index, NTproBNP N-terminal pro-B-type natriuretic peptide, NYHA New York Heart Association functional class, $R_{N}{ }^{2}$ Nagelkerke $R^{2}$ progression and outcome in patients with $\mathrm{AR}[1,4,5$, $22,23]$. The EF of the AR group in our study increased from baseline to follow-up. This EF increase was a function of reductions in EDV, ESV, and SV, and might primarily be a marker of reversed LV remodeling in these patients. We also measured GLS, which has previously been shown predictive of mortality, the need for surgery, and LV systolic dysfunction in patients with $\operatorname{AR}[9,10$, $24,25]$. We found baseline average GLS values in the same range (i.e., around $-19 \%$ ) as those reported in a recent study of patients with AR, where lower absolute values were found to be associated with increased mortality [9]. However, we did not find an association between GLS and the composite outcome of impaired LV functional and structural recovery following surgery. Furthermore, GLS did not change significantly from baseline to follow-up. This may be partly explained by the load dependency of GLS, where an increase in SV and concomitant increase in EDV, as seen in AR, will have opposing effects on GLS [26, 27]. The same observation was made by Vollema et al., who suggested that the finding was related to the Frank-Starling mechanism; hence, a reduction in preload will lead to a decrease in myocardial contraction force and consequently GLS [28]. The interaction between GLS and loading conditions was also evident in a study of 47 patients with AR undergoing valve surgery, in whom the absolute GLS decreased after surgery, whereas the normalized GLS calculated as the GLS/EDV ratio increased, primarily driven by a reduction in EDV after surgery [29].

We further analyzed the relationship between changes in LAS and GLS, finding that the latter had a moderate linear correlation with changes in LASr and LAScd. This finding confirms that GLS and LAS partly interact during systole, probably because systolic atrioventricular plane movement affects LA strain in segments close to the mitral annulus.

\section{Diastolic LV function and LA function}

LV-LA interaction is complex in patients with AR. If the LV is compliant, increased preload results in 
Table 5 Multiple logistic regression analysis and LRT showing models that best predicted impaired LV functional and structural recovery in patients undergoing surgery for aortic regurgitation

\begin{tabular}{|c|c|c|c|c|c|c|c|}
\hline & Model $p$ & Accuracy (\%) & Sensitivity (\%) & Specificity (\%) & $R_{\mathrm{N}}^{2}$ & AUC (95\% Cl) & LRT $p$ \\
\hline ESVi & $<0.001$ & 69 & 59 & 77 & 0.28 & $0.78(0.66-0.88)$ & \\
\hline \multicolumn{8}{|c|}{ Variable added to ESVi } \\
\hline Age & $<0.001$ & 69 & 59 & 77 & 0.33 & $0.78(0.66-0.90)$ & 0.11 \\
\hline LAScd & $<0.001$ & 70 & 65 & 74 & 0.42 & $0.83(0.72-0.93)$ & 0.006 \\
\hline LASr & $<0.001$ & 68 & 61 & 74 & 0.33 & $0.80(0.69-0.91)$ & 0.10 \\
\hline Stroke work & 0.001 & 68 & 56 & 77 & 0.29 & $0.77(0.65-0.89)$ & 0.70 \\
\hline
\end{tabular}

AUC Area under the ROC curve, ESVi End-systolic volume index, LAScd Left atrial strain conduit phase, LASr Left atrial strain reservoir phase, $L R T$ likelihood ratio test, $R_{N}{ }^{2}$, Nagelkerke $R^{2}$, Zva Valvulo-arterial impedance

increased EDV without any increase in diastolic LV filling pressure. In the latter disease stages, there is an increase in the fibrous content of the LV myocardium, leading to increased stiffness, which contributes to increased LV filling pressure and a subsequent increase in mean LA pressure and LA volume; at some point, this will become associated with symptom development [30, 31]. Previous studies have revealed a significant interaction between LA phasic function and LV diastolic and systolic function in patients with aortic stenosis, coronary artery disease, and heart failure, but not in severe AR. In cases of aortic stenosis, the LAScd was correlated with indices of increased LV filling pressure, while LASct was correlated with aortic valve area [32]. In patients with coronary artery disease and heart failure, LASrand to a lesser extent, LASct-correlate with LV enddiastolic pressure, LA pressure, and NTproBNP levels [32-35]. This is consistent with our findings in patients with AR, where LASr and LAScd correlated with NTproBNP levels.

In a previous study of patients with DD and preserved $\mathrm{EF}$, mild DD was associated with reductions in LAScd and LASr and an increase in LASct, whereas all components of LA phasic function were reduced in patients with higher degrees of DD [36]. We also found an association between LA phasic function and DD in patients with AR, with lower LASr and LASct values in patients with DD grades 2 or 3 compared with those with DD grades 0 or 1 . In parallel, the NTproBNP concentration was 2.3 times higher in our patients with DD grades 2 or 3 , suggesting that there was, indeed, a difference in LV filling pressure between the groups.

The reduced preoperative LASct in our patients with AR with DD grade $>1$ indicates a diminished active atrial contraction, consistent with a previous report showing that LA contraction force is lower in patients with AR than in those with aortic stenosis [37]. In a mixed group of patients with AR or aortic stenosis, LASct was the first of the phasic LA function components to be altered in moderate valvular disease, whereas both LASct and LASr were reduced in patients with severe aortic valvular disease with pulmonary hypertension [38]. The reduction in LA contractile function in patients with AR might be explained by an increased afterload imposed on the left atrium caused by a combination of competitive filling of the left ventricle from the aorta and increased stiffness of the LV wall. LA phasic function is also directly influenced by preload, as demonstrated in a study of healthy volunteers, where all LAS components were reduced following an acute preload reduction [39]. The reduction in volume load and LV preload following aortic valve surgery improved LAS components in our patients with AR. The variable that best predicted the composite outcome of impaired LV recovery was the well-established measure of end-systolic LV dimension [4]. However, LAScd added significantly to the prediction, suggesting that LAScd has additive prognostic value for patients with AR. A high prevalence of DD in patients with severe AR, and its adverse impact on postoperative cardiac function, has been reported previously [40]. In our study, moderate or advanced DD was found at baseline in one-third of the patients with AR. Although DD improved postoperatively, DD grades 2 or 3 persisted in some patients. This observation may be related to residual LV fibrosis in these patients. In a previous study, myocardial fibrosis and invasively assessed LV diastolic stiffness were increased in AR patients preoperatively, and remained increased after AVR [41].

\section{Limitations}

The echocardiographic assessment of LV diastolic function and filling pressure in patients with AR remains challenging. There are limited data regarding the accuracy of current criteria for the assessment of increased LV filling pressures in these patients [12]. Nevertheless, in our patients with AR, a significant difference was observed in the NTproBNP concentration in patients with DD grades 2 or 3 compared with patients with DD grades 0 or 1 , supporting the validity of the integrative approach to differentiate between normal and increased filling pressures. 
Our control group consisted of patients free from significant aortic valve disease, who underwent open thoracic surgery for TAA. Our goal was to assess whether cardiac surgery per se would induce changes in LV function or LAS. Although we cannot exclude small changes in loading conditions due to the surgical correction, we could not demonstrate any significant changes in LV or LA volumes or function in the control group. Therefore, we believe that the volumetric and functional changes observed in our patients with AR were not significantly affected by the trauma of surgery per se.

Our relatively short follow-up time precluded use of hard outcomes such as mortality or admissions for recurrent heart failure. Furthermore, we used a composite variable to define impaired LV function and structure, which may limit the generalizability of the results. Thus, our study should be considered a hypothesis-generating one. The prognostic implications of LAS in patients with AR warrants validation with larger cohorts with additional clinical outcome variables.

\section{Conclusions}

One-third of patients with chronic severe AR had signs of impaired LV diastolic function. Aortic valve surgery improved diastolic LV function, decreased LV and LA volumes, and increased LA reservoir and contractile function. We found that LA strain components have an incremental prognostic value to the well-established LV end-systolic dimension for the prediction of impaired LV functional and structural recovery following aortic valve surgery. However, further research in this area with larger, longer-term follow-up studies with hard endpoints will be needed to establish the role of LAS in the preoperative evaluation of patients with AR.

\begin{abstract}
Abbreviations
ANOVA: Analysis of variance; AR: Aortic regurgitation; AUC: Area under the curve; AVR: Aortic valve replacement; BSA: Body surface area; Cl: Confidence interval; DD: Diastolic dysfunction; EDV: End-diastolic volume; ESV: Endsystolic volume; EF: Ejection fraction; GLS: Global longitudinal strain; ICC: Intraclass correlation coefficient; LA: Left atrial; LAS: Left atrial strain; LAScd: Left atrial strain conduit phase; LASct: Left atrial strain contraction phase; LASr: Left atrial strain reservoir phase; LAVi: Left atrial volume index; LRT: Likelihood ratio test; LV: Left ventricular; LVMi: Left ventricular mass index; NYHA: New York Heart Association; NPX: Normalized protein expression; NTproBNP: N-terminal pro-B-type natriuretic peptide; ROC: Receiver operating characteristic; $\mathrm{R}_{N}{ }^{2}$ : Nagelkerke $R^{2}$; SV: Stroke volume; TAA: Thoracic aortic aneurysm; Zva: Valvulo-arterial impedance
\end{abstract}

\section{Acknowledgements}

We would like to acknowledge Jonas Selling, Senior Statistician, for valuable statistical support, and Mahmood Farasati and Kamel Ramak for the echocardiographic examinations.

\section{Authors' contributions}

J.J. acquired and analyzed data and drafted and finalized the manuscript. A.l. acquired and analyzed data. J.P. revised the manuscript. P.E. acquired data and revised the manuscript. A.F.C. contributed to the conception and design, data acquisition and analysis, and manuscript revision. M.J.E. contributed to the conception and design, data analysis, supervision, and manuscript revision. K.C. contributed to the conception and design, supervision, data analysis, and manuscript revision. All authors read and approved the final manuscript.

\section{Funding}

The study was supported by the Swedish Heart Lung Foundation, the Swedish Research Council, grants from the Swedish state under an agreement between the Swedish government and the county councils (the ALF agreement), and a donation from Fredrik Lundberg. The funders had no influence on the study design, data collection, analysis, data interpretation, or writing of the manuscript. Open Access funding provided by Karolinska Institute.

\section{Availability of data and materials}

The data that support the study findings are available from the corresponding author upon reasonable request.

\section{Ethics approval and consent to participate}

Informed, written consent was obtained from all study participants, and the study was approved by the Regional Ethics Review Board in Stockholm.

\section{Consent for publication}

Not applicable.

\section{Competing interests}

The authors declare that they have no conflict of interest.

\section{Author details}

${ }^{1}$ Department of Molecular Medicine and Surgery, Karolinska Institutet, Stockholm, Sweden. ²Department of Clinical Physiology, Södersjukhuset, Stockholm, Sweden. ${ }^{3}$ Department of Clinical Physiology, Karolinska University Hospital, 17176 Stockholm, Sweden. ${ }^{4}$ Division of Cardiovascular Medicine, Department of Medicine Solna, Karolinska University Hospital, Karolinska Institutet, Stockholm, Sweden. ${ }^{5}$ Unit of Cardiothoracic Surgery, Karolinska University Hospital, Stockholm, Sweden. ${ }^{6}$ Departments of Molecular and Clinical Medicine, Gothenburg University, and Clinical Physiology,

Sahlgrenska University Hospital, Gothenburg, Sweden.

Received: 2 November 2020 Accepted: 31 January 2021

Published online: 14 February 2021

\section{References}

1. Dujardin KS, Enriquez-Sarano M, Schaff HV, Bailey KR, Seward JB, Tajik AJ. Mortality and morbidity of aortic regurgitation in clinical practice. A longterm follow-up study. Circulation. 1999;99:1851-7.

2. Bonow RO, Lakatos E, Maron BJ, Epstein SE. Serial long-term assessment of the natural history of asymptomatic patients with chronic aortic regurgitation and normal left ventricular systolic function. Circulation. 1991; 84:1625-35.

3. Chaliki HP, Mohty D, Avierinos JF, Scott CG, Schaff HV, Tajik AJ, et al. Outcomes after aortic valve replacement in patients with severe aortic regurgitation and markedly reduced left ventricular function. Circulation. 2002:106:2687-93.

4. Baumgartner H, Falk V, Bax JJ, De Bonis M, Hamm C, Holm PJ, et al. 2017 ESC/EACTS guidelines for the management of valvular heart disease. Eur Heart J. 2017;38:2739-91.

5. Nishimura RA, Otto CM, Bonow RO, Carabello BA, Erwin JP 3rd, Guyton RA, et al. 2014 AHA/ACC guideline for the management of patients with valvular heart disease: executive summary: a report of the American College of Cardiology/American Heart Association task force on practice guidelines. J Am Coll Cardiol. 2014;63:2438-88.

6. Rahhab Z, El Faquir N, Tchetche D, Delgado V, Kodali S, Mara Vollema E, et al. Expanding the indications for transcatheter aortic valve implantation. Nat Rev Cardiol. 2020;17:75-84.

7. de Meester C, Gerber BL, Vancraeynest D, Pouleur AC, Noirhomme P, Pasquet $A$, et al. Do guideline-based indications result in an outcome penalty for patients with severe aortic regurgitation? JACC CardiovasC Imaging. 2019;12:2126-38.

8. Yang LT, Michelena HI, Scott CG, Enriquez-Sarano M, Pislaru SV, Schaff HV, et al. Outcomes in chronic Hemodynamically significant aortic regurgitation and limitations of current guidelines. J Am Coll Cardiol. 2019;73:1741-52. 
9. Alashi A, Khullar T, Mentias A, Gillinov AM, Roselli EE, Svensson LG, et al. Long-term outcomes after aortic valve surgery in patients with asymptomatic chronic aortic regurgitation and preserved LVEF: impact of baseline and follow-up global longitudinal strain. JACC Cardiovasc Imaging. 2020;13:12-21.

10. Olsen NT, Sogaard P, Larsson HB, Goetze JP, Jons C, Mogelvang R, et al. Speckle-tracking echocardiography for predicting outcome in chronic aortic regurgitation during conservative management and after surgery. JACC Cardiovasc Imaging. 2011;4:223-30.

11. Zaid RR, Barker CM, Little SH, Nagueh SF. Pre- and post-operative diastolic dysfunction in patients with valvular heart disease: diagnosis and therapeutic implications. J Am Coll Cardiol. 2013;62:1922-30.

12. Nagueh SF, Smiseth OA, Appleton CP, Byrd BF 3rd, Dokainish H, Edvardsen $T$, et al. Recommendations for the evaluation of left ventricular diastolic function by echocardiography: an update from the American Society of Echocardiography and the European Association of Cardiovascular Imaging. Eur Heart J Cardiovasc Imaging. 2016;17:1321-60.

13. Gan GCH, Ferkh A, Boyd A, Thomas L. Left atrial function: evaluation by strain analysis. Cardiovasc Diagn Ther. 2018;8:29-46.

14. Meimoun P, Djebali M, Botoro T, Djou Md U, Bidounga H, Elmkies F, et al. Left atrial strain and distensibility in relation to left ventricular dysfunction and prognosis in aortic stenosis. Echocardiography. 2019;36:469-77.

15. Candan O, Ozdemir N, Aung SM, Hatipoglu S, Karabay CY, Guler A, et al. Atrial longitudinal strain parameters predict left atrial reverse remodeling after mitral valve surgery: a speckle tracking echocardiography study. Int J Card Imaging. 2014;30:1049-56.

16. Cameli M, Mandoli GE, Loiacono F, Dini FL, Henein M, Mondillo S. Left atrial strain: a new parameter for assessment of left ventricular filling pressure. Heart Fail Rev. 2016;21:65-76.

17. Jackson V, Petrini J, Caidahl K, Eriksson MJ, Liska J, Eriksson P, et al. Bicuspid aortic valve leaflet morphology in relation to aortic root morphology: a study of 300 patients undergoing open-heart surgery. Eur J Cardiothorac Surg. 2011;40:e118-24.

18. Lang RM, Badano LP, Mor-Avi V, Afilalo J, Armstrong A, Ernande L, et al. Recommendations for cardiac chamber quantification by echocardiography in adults: an update from the American Society of Echocardiography and the European Association of Cardiovascular Imaging. Eur Heart J Cardiovasc Imaging. 2015;16:233-70.

19. de Simone G, Chinali M, Galderisi M, Benincasa M, Girfoglio D, Botta I, et al. Myocardial mechano-energetic efficiency in hypertensive adults. J Hypertens. 2009;27:650-5.

20. Badano LP, Kolias TJ, Muraru D, Abraham TP, Aurigemma G, Edvardsen T, et al. Standardization of left atrial, right ventricular, and right atrial deformation imaging using two-dimensional speckle tracking echocardiography: a consensus document of the EACVI/ASE/industry task force to standardize deformation imaging. Eur Heart J Cardiovasc Imaging 2018;19:591-600.

21. Taniguchi K, Nakano S, Kawashima Y, Sakai K, Kawamoto T, Sakaki S, et al. Left ventricular ejection performance, wall stress, and contractile state in aortic regurgitation before and after aortic valve replacement. Circulation. 1990;82:798-807.

22. Verseckaite R, Mizariene V, Montvilaite A, Auguste I, Bieseviciene M, Laukaitiene J, et al. The predictive value of left ventricular myocardium mechanics evaluation in asymptomatic patients with aortic regurgitation and preserved left ventricular ejection fraction. A long-term speckle-tracking echocardiographic study. Echocardiography. 2018;35:1277-88.

23. Barbieri A, Benfari G, Giubertoni E, Manicardi M, Bursi F, Rossi A, et al. Degree of left ventricular dilatation at end-diastole: correlation and prognostic utility of quantitative volumes by 2D-echocardiography versus linear dimensions in patients with asymptomatic aortic regurgitation. Echocardiography. 2020;37:1336-44.

24. Ewe SH, Haeck ML, Ng AC, Witkowski TG, Auger D, Leong DP, et al. Detection of subtle left ventricular systolic dysfunction in patients with significant aortic regurgitation and preserved left ventricular ejection fraction: speckle tracking echocardiographic analysis. Eur Heart J Cardiovasc Imaging. 2015;16:992-9.

25. Alashi A, Mentias A, Abdallah A, Feng K, Gillinov AM, Rodriguez LL, et al. Incremental prognostic utility of left ventricular global longitudinal strain in asymptomatic patients with significant chronic aortic regurgitation and preserved left ventricular ejection fraction. JACC Cardiovasc Imaging. 2018; 11:673-82.
26. Burns AT, La Gerche A, D'Hooge J, Maclsaac Al. Prior DL. Left ventricular strain and strain rate: characterization of the effect of load in human subjects. Eur J Echocardiogr. 2010;11:283-9.

27. Weidemann F, Jamal F, Sutherland GR, Claus $P$, Kowalski $M$, Hatle $L$, et al. Myocardial function defined by strain rate and strain during alterations in inotropic states and heart rate. Am J Physiol Heart Circ Physiol. 2002;283: H792-9.

28. Vollema EM, Singh GK, Prihadi EA, Regeer MV, Ewe SH, Ng ACT, et al. Time course of left ventricular remodelling and mechanics after aortic valve surgery: aortic stenosis vs aortic regurgitation. Eur Heart J Cardiovasc Imaging. 2019;20:1105-11.

29. Smedsrud MK, Pettersen E, Gjesdal O, Svennevig JL, Andersen K, Ihlen H, et al. Detection of left ventricular dysfunction by global longitudinal systolic strain in patients with chronic aortic regurgitation. J Am Soc Echocardiogr. 2011;24:1253-9.

30. Borer JS, Truter S, Herrold EM, Falcone DJ, Pena M, Carter JN, et al. Myocardial fibrosis in chronic aortic regurgitation: molecular and cellular responses to volume overload. Circulation. 2002;105:1837-42.

31. Braunwald E, Frahm CJ. Studies on Starling's law of the heart: IV. Observations on the hemodynamic functions of the left atrium in man. Circulation. 1961;24:633-43.

32. O'Connor K, Magne J, Rosca M, Pierard LA, Lancellotti P. Impact of aortic valve stenosis on left atrial phasic function. Am J Cardiol. 2010;106:1157-62.

33. Wakami K, Ohte N, Asada K, Fukuta H, Goto T, Mukai S, et al. Correlation between left ventricular end-diastolic pressure and peak left atrial wall strain during left ventricular systole. J Am Soc Echocardiogr. 2009;22:847-51.

34. Lundberg A, Johnson J, Hage C, Back M, Merkely B, Venkateshvaran A, et al. Left atrial strain improves estimation of filling pressures in heart failure: a simultaneous echocardiographic and invasive haemodynamic study. Clin Res Cardiol. 2019:108:703-15.

35. Loar RW, Pignatelli RH, Morris SA, Colquitt JL, Feagin DK, Denfield SW, et al. Left atrial strain correlates with elevated filling pressures in pediatric heart transplantation recipients. J Am Soc Echocardiogr. 2020;33:504-11.e1.

36. Singh A, Addetia K, Maffessanti F, Mor-Avi V, Lang RMLA. Strain for categorization of LV diastolic dysfunction. JACC Cardiovasc Imaging. 2017; 10:735-43.

37. Cioffi G, Stefenelli C. Comparison of left ventricular geometry and left atrial size and function in patients with aortic stenosis versus those with pure aortic regurgitation. Am J Cardiol. 2002;90:601-6.

38. Salas-Pacheco JL, Avila-Vanzzini N, Eugenia RM, Arias-Godinez JA. Left atrium function by $2 \mathrm{D}$ speckle tracking in aortic valve disease. Echocardiography. 2016;33:1828-34.

39. Genovese D, Singh A, Volpato V, Kruse E, Weinert L, Yamat M, et al. Load dependency of left atrial strain in Normal subjects. J Am Soc Echocardiogr. 2018;31:1221-8.

40. Ma W, Zhang W, Shi W, Kong Y, Ma X. Left ventricular diastolic function after aortic valve replacement for chronic aortic regurgitation. Ann Thorac Surg. 2018;106:24-9.

41. Villari B, Sossalla S, Ciampi Q, Petruzziello B, Turina J, Schneider J, et al. Persistent diastolic dysfunction late after valve replacement in severe aortic regurgitation. Circulation. 2009;120:2386-92.

\section{Publisher's Note}

Springer Nature remains neutral with regard to jurisdictional claims in published maps and institutional affiliations.

\section{Ready to submit your research? Choose BMC and benefit from:}

- fast, convenient online submission

- thorough peer review by experienced researchers in your field

- rapid publication on acceptance

- support for research data, including large and complex data types

- gold Open Access which fosters wider collaboration and increased citations

- maximum visibility for your research: over $100 \mathrm{M}$ website views per year

At $\mathrm{BMC}$, research is always in progress.

Learn more biomedcentral.com/submission 